\title{
Interfaces and internal layers in a turbulent boundary layer
}

\author{
Jerke Eisma, ${ }^{\text {a) }}$ Jerry Westerweel, ${ }^{\text {b) }}$ Gijs Ooms, ${ }^{\text {c) }}$ and Gerrit E. Elsinga ${ }^{d)}$ \\ Laboratory for Aero and Hydrodynamics, Delft University of Technology, \\ 2628 CD Delft, The Netherlands
}

(Received 26 February 2015; accepted 22 April 2015; published online 14 May 2015)

\begin{abstract}
New experimental research is presented on the characteristics of interfaces and internal shear layers that are present in a turbulent boundary layer (TBL). The turbulent/non-turbulent (T/NT) interface at the outer boundary of the TBL shows the presence of a finite jump in streamwise velocity and is characterised by a thin shear layer. It appears that similar layers of high shear occur also within the TBL which separate regions of almost uniform momentum. It turns out that they exhibit similar characteristics as the external T/NT interface. Furthermore, the spatial growth rate of the TBL, that is derived from theoretical analysis, can be correctly predicted from a momentum balance near the external T/NT interface. Similarly, the entrainment velocities for the average internal layers have been determined. Results indicate that internal layers move slower in the vicinity of the wall, whereas they move faster than the large scale boundary layer growth rate in the outer region of the TBL. It is believed that shear layers bound large scale flow regions of approximately uniform momentum. Hence, the entrainment velocities of these internal layers may be interpreted as growth rates of the large scale motions in a TBL. ( 2015 AIP Publishing LLC. [http://dx.doi.org/10.1063/1.4919909]
\end{abstract}

\section{INTRODUCTION}

In many fluid flows, such as jets, wakes, and boundary layers, the turbulent flow is enclosed by an irrotational non-turbulent flow domain. The pioneering work of Corssin and Kistler ${ }^{1}$ has shown that there exists a thin layer that separates the turbulent from the non-turbulent flow, with strong jumps in vorticity and/or velocity across these layers. Previous research has recognized the significance of these interfaces on the overall dynamics of the turbulent flow. ${ }^{1-9}$ Knowledge on physical mechanisms occurring at these interfaces is especially important as a number of processes take place at or in close vicinity of the interface, e.g., it governs the overall growth of the turbulent flow region as entrainment of non-turbulent fluid into the turbulent flow occurs across this interface. Previous research on the turbulent/non-turbulent (T/NT) interface has focused on the definition of the interface and on the physical mechanism of entrainment at the interface. The usual way to detect interfaces is using threshold based methods,${ }^{10}$ giving rise to some arbitrariness in the exact definition of the interface. However, the consensus is that there exists a T/NT interface that is bounded by a viscous superlayer that separates the different flow regions. ${ }^{10,11}$ Furthermore, entrainment over the T/NT interface has been researched for several unbounded flows, ${ }^{3,4,7,8}$ where it has been shown that the entrainment process at the T/NT interface is mainly dominated by a small scale process ("nibbling"). Chauhan et al. ${ }^{12}$ have shown that entrainment over the T/NT interface in a turbulent boundary layer (TBL) is characterized by small scale "nibbling," whereas large scales present at the interface convolute the interface. As the large scales govern the overall entrainment, the small scale motions turn non-turbulent fluid by viscous diffusion into a turbulent state. The characteristics of the

\footnotetext{
${ }^{a)}$ H.E.Eisma@tudelft.nl

b) J.Westerweel@tudelft.nl

c) G.Ooms@tudelft.nl

d) G.E.Elsinga@ @udelft.nl
} 
$\mathrm{T} / \mathrm{NT}$ interface in a TBL have been studied before by Ishihara et al. ${ }^{13}$ by direct numerical simulation (DNS). A sharp drop in conditional two-point correlations was observed indicating that the T/NT interface acts as a barrier to external non-turbulent fluctuations.

Thin layers, as described by Corssin and Kistler, ${ }^{1}$ however, are not only found in the region that separates the turbulent flow from the non-turbulent region but also between layers of different turbulent intensities. ${ }^{2,14}$ Recently, internal layers with similar characteristics as a T/NT interface within homogeneous isotropic turbulence were reported by Ishihara et al. ${ }^{5,6}$ According to Robinson ${ }^{15}$ (and references therein), internal shear layers have been observed throughout TBLs as well, even in the outer region of the flow. For instance, Blackwelder and Kovasznay ${ }^{16}$ showed that significant shear layers were observed upstream of large scale motions based on space-time correlations of hotwire data in a TBL. Meinhart and Adrian, ${ }^{17}$ their Figure 2 and Adrian et al. ${ }^{18}$ and their Figure 17 visually observed internal thin shear layers in a TBL that separated large scale regions with nearly uniform momentum. These large scale regions were also observed by others, ${ }^{19}$ who found that these structures persist over long streamwise distances. The dynamical significance of these large scale structures has been elucidated in recent years with the evolution of new experimental measurement techniques. ${ }^{20}$ It was found that these large scale regions are statistically relevant by carrying a significant amount of kinetic energy and have an important contribution to the Reynolds shear stresses while only occupying a small portion of the total flow volume. ${ }^{21,22}$ Not only instantaneous observations have been made of these internal shear layers, but also linear stochastic estimation by Christensen and Adrian ${ }^{23}$ [their Figure 4] and Hambleton et al. ${ }^{21}$ [their Figure 6] show clearly structures separating two large-scale flow regions. Moreover, it turns out that layered structures were found to be characteristic of the mean structure associated with the principal strain in different turbulent flows by Elsinga and Marusic, ${ }^{24}$ which therefore can be considered to be typical for turbulent flow fields. Wei et al. ${ }^{25}$ have shown that the flow around the shear layers in a TBL exhibits a scaling related to the macro-scales in a TBL. This indicates that the shear layer is closely related to large scales present in the flow. Although the relevance of these interfaces and internal layers on the overall dynamics of the flow has been suggested for unbounded turbulent flows by several authors, ${ }^{2,7}$ only minor attention has been paid to the characteristics of these internal layers present in wall bounded turbulent flows.

In this paper, we examine the characteristics of the T/NT interface and internal layers that are observed in the instantaneous flow fields in experimental data of a turbulent boundary layer. In our present work, we define internal layers as regions of high shear. It should be noted that internal layers are not solely characterized by regions of intense shear; there may also be internal layers without significant shear. ${ }^{2}$ Internal layers and the T/NT interface are shown to have similar characteristics and can be described by the same theoretical framework. By quantifying the entrainment over the bounding shear layers, growth rates of the associated large scale regions can be estimated, which is analogous to the overall TBL growth by the entrainment across the T/NT interface. This sheds new light on the growth mechanisms of large scale structures in a TBL, which are bounded by these layers.

The work in this paper is arranged as follows. Section II describes the experimental details of the experiment including the global boundary layer properties. Section III describes the different detection criteria that are employed for the T/NT interface and the internal layers. Section IV describes the model that is employed to derive the boundary entrainment velocity for internal shear layers as well as the external T/NT interface. In Sec. V, the conditional sampled profiles around these layers are discussed and the boundary entrainment velocity derived from these statistics is shown. Section VI discusses the implications of the previous sections (mainly Sec. V) in relation to previous obtained results. Finally, Sec. VII summarizes and draws final conclusions.

\section{EXPERIMENTAL SETUP}

The experiment is performed in the water tunnel at the Laboratory for Aero- and Hydrodynamics at the Delft University of Technology. It has a test section with dimensions $L \times W \times H$ $=5 \times 0.6 \times 0.6 \mathrm{~m}^{3}$. Boundary layer measurements were done at one of the side walls, approximately $3.5 \mathrm{~m}$ downstream of a boundary layer trip, at a free-stream velocity of $U_{\infty}=0.73 \mathrm{~m} / \mathrm{s}$. 
TABLE I. Summary of the boundary layer properties.

\begin{tabular}{llcccc}
\hline \hline$U_{\infty}$ & $0.73 \mathrm{~m} / \mathrm{s}$ & $\theta$ & $8.7 \mathrm{~mm}$ & $R e_{\tau}$ & 2053 \\
$\delta_{99}$ & $74 \mathrm{~mm}$ & $u_{\tau}$ & $26.5 \mathrm{~mm} / \mathrm{s}$ & $H=\delta^{*} / \theta$ & 1.31 \\
$\delta^{*}$ & $11.3 \mathrm{~mm}$ & $\operatorname{Re}_{\theta}$ & 6578 & $c_{f}$ & $2.7 \times 10^{-3}$ \\
\hline \hline
\end{tabular}

At this location, the boundary layer thickness was $\delta_{99}=74 \mathrm{~mm}$, where $\delta_{99}$ is the wall distance where the velocity has reached $99 \%$ of the free-stream value. The corresponding momentum and displacement thickness are $\theta=8.7 \mathrm{~mm}$ and $\delta^{*}=11.3 \mathrm{~mm}$, resulting in a shape factor $H=1.31$, which is a typical value for a zero-pressure gradient TBL. The Reynolds number based on the momentum thickness is $R e_{\theta}=6578$. Finally, the friction velocity $u_{\tau}$ was derived from a Clauser plot technique $\left(u_{\tau}=26.5 \mathrm{~mm} / \mathrm{s}\right)$. The main properties of the boundary layer are summarized in Table I.

For the stereoscopic particle image velocimetry (PIV) measurements, the flow is seeded with $10 \mu \mathrm{m}$ buoyant neutral tracer particles (Sphericell). A streamwise-wall-normal plane is illuminated with a twin-cavity double pulsed Nd:YAG laser (Spectra-Physics Quanta Ray). The thickness of this light sheet was $1 \mathrm{~mm}$. The particle images were recorded using two high resolution CCD cameras with a $4872 \times 3248$ pixel format (Image LX 16M, LaVision) which were equipped with a Micro-Nikkor F105 mm objective operating at an aperture number $f_{\#}=8$. The resulting field of view was $1.35 \delta_{99}$ in streamwise direction and $1.87 \delta_{99}$ in wall-normal direction. Calibration, data acquisition, and post-processing were performed with a commercial software package (Davis 8.1, LaVision). The PIV images are interrogated with a multi-pass interrogation technique, where the final interrogation windows have a size of $24 \times 24$ pixels with $75 \%$ overlap, corresponding to a spatial resolution based on the window size of 19.9 viscous wall units $\left(u_{\tau} / v\right)$. This is approximately 3-4 times the Kolmogorov length scale in the outer layer, ${ }^{26}$ hence, the current measurement can be regarded as fully resolved. Individual spurious vectors are detected using a median test ${ }^{27}$ and replaced by linear interpolation. In total, 1824 in-plane instantaneous velocity fields were captured at a frame rate of $0.77 \mathrm{~Hz}$ to obtain sufficiently converged first and second order statistics.

The velocity profile in inner scaling is shown in Figure 1(a). Reliable instantaneous data could be obtained for distances of 25 or more viscous wall units from the wall. Good agreement with data from literature is observed in the outer layer of the TBL. The outer regions of the RMS profiles shown in Figure 1(b) have been corrected for the PIV measurement noise. An estimate of the noise contribution to the RMS values was obtained from the method described by Poelma et $a l .{ }^{28}$ It should be noted that Klebanoff ${ }^{29}$ adopts a different definition for the boundary layer thickness. Based on the intermittency curves (not shown here), it is estimated that this is approximately given as $\delta_{K l e b} \approx 1.3 \delta_{99}$; this has been corrected for in Figure 1(b). However, Figure 1(b)
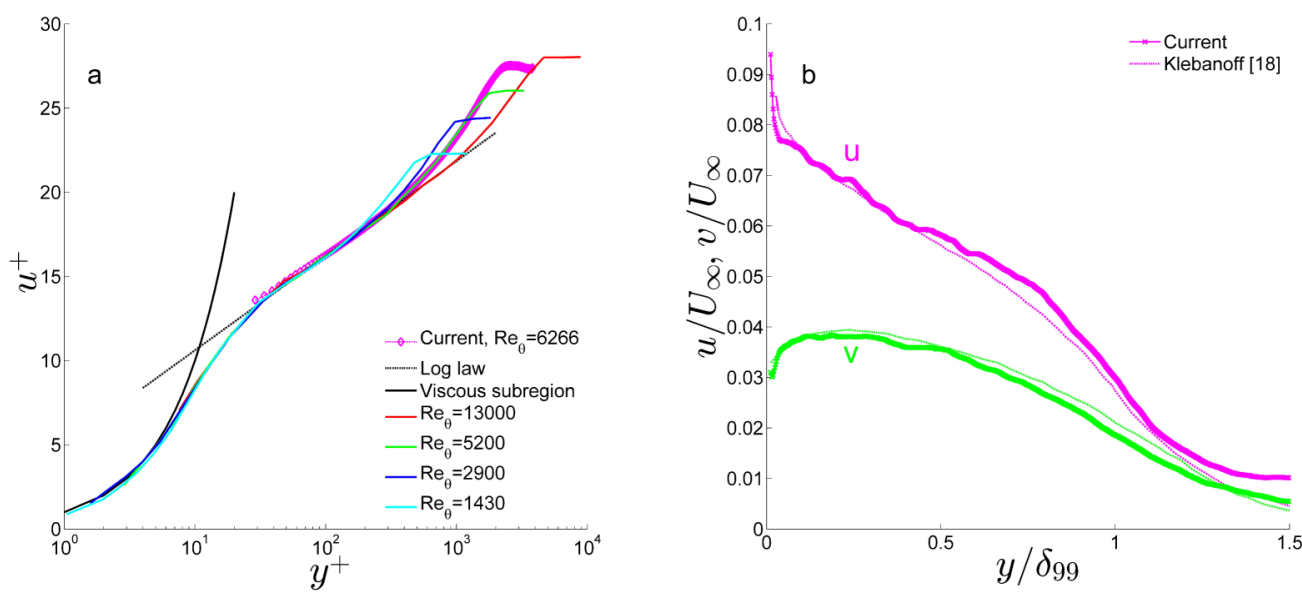

FIG. 1. (a) The velocity profile in inner scaling compared with data from DeGraaff and Eaton. ${ }^{32}$ (b) The RMS profiles of the streamwise and wall-normal components of the velocity compared with data from Klebanoff. ${ }^{29}$ 
still shows that the RMS profiles are slightly higher in the free stream compared to reference data from Klebanoff. ${ }^{29}$ This may be attributed to the higher freestream turbulence intensity in the facility as well as to the uncertainty in determining the boundary layer thickness $\delta_{99}$. The value for the free-stream turbulence intensity is found to be below $1 \% U_{\infty}$. This is slightly higher compared to the turbulence intensity as reported by Schröder et $a l .{ }^{30}$ who performed PIV measurements in the same facility. Finally, the current measurements are subject to a very small favourable pressure gradient. Independent pitot measurements indicate the acceleration parameter, $K=2 \times 10^{-8}$. As shown by Joshi et al. ${ }^{31}$ effects on the turbulence structure in a boundary layer become noticeable at $K=10^{-6}$. Hence, the pressure gradient will have a negligible influence on the results discussed here.

\section{LAYER DETECTION AND GEOMETRICAL CHARACTERISTICS}

\section{A. T/NT interface}

The T/NT interface is detected using a method that relies on the out-of-plane vorticity $\Omega_{z}$, which is found to be the dominant component at the interface. ${ }^{33}$ Gaussian smoothing, characterized by a filter width of $\sigma \approx 10 y^{+}$, of the velocity field is performed after which the available velocity gradients are calculated using a second order least squares difference scheme. ${ }^{34}$ Care is taken to ensure that the filter width is smaller than the Taylor micro-scale $\lambda_{T} \approx 180 y^{+}$, to prevent affecting the relevant flow scales. Subsequent conditional sampling is performed on the raw unfiltered velocity fields. This approach is justified because the thickness of the interfaces $\delta_{w}$ is commonly regarded to be of the order of $\lambda_{T}$ for flows with a mean shear. ${ }^{7}$ Detection of the T/NT interface was performed using the vorticity method as proposed by Holzner et al. ${ }^{8}$ which proves to be effective in detecting the T/NT interface. A noise level for the instantaneous vorticity is estimated by taking the RMS values of $\Omega_{z}$ in the non-turbulent part of the flow (i.e., $y / \delta_{99} \geq 1.5$ ), which is approximately $2.7 \mathrm{~s}^{-1}$. A threshold of three times this level effectively separates the irrotational non-turbulent region from the turbulent region. The T/NT interface is then defined as the set of outermost points that exceed this threshold. The average interface position is found to be at $\left\langle\delta_{\text {int }}\right\rangle \approx 0.9 \delta_{99}$ (see Figure 2) which is somewhat higher than the result of Corssin and $\operatorname{Kistler}^{1}$ who found $\left\langle\delta_{\text {int }}\right\rangle \approx 0.80 \delta_{99}$.

\section{B. Internal layers}

In order to detect the internal layers, the triple decomposition method as proposed by Kolár ${ }^{35}$ is applied. This decomposition was originally introduced to provide a more robust description of

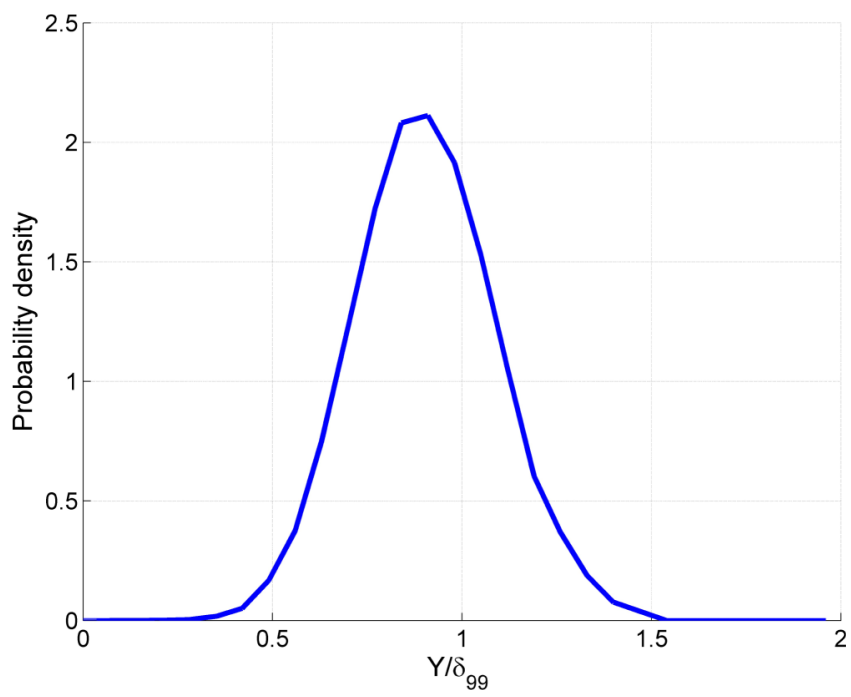

FIG. 2. Probability density function of the location of the interface $y_{i}$. The average interface location is found at $y_{i}=0.9 \delta_{99}$. 
vortices in turbulent flows. However, the method can also be used to detect shear layers. ${ }^{36}$ The velocity gradient tensor $\nabla \mathbf{u}$ is decomposed into three parts,

$$
\nabla \mathbf{u}=(\nabla \mathbf{u})_{R R}+(\nabla \mathbf{u})_{S H}+(\nabla \mathbf{u})_{E L},
$$

where $R R, S H$, and $E L$ denote the rigid-body rotation, shear, and elongation components of the velocity gradient tensor, respectively. This decomposition only applies to an appropriate reference frame, the so-called basic reference frame (BRF). In order to derive the shear component $(\nabla \mathbf{u})_{S H}$ in Eq. (1), the following steps are performed: first, the $2 \mathrm{D}$ velocity gradient tensor $\nabla \mathbf{u}$ and the strain rate tensor (i.e., the symmetric part of $\nabla \mathbf{u}$ ) are computed; second, the eigenvectors of the strain rate tensor are found resulting in the principal axes; and third, $\nabla \mathbf{u}$ is expressed in the BRF with orthogonal axes that are rotated $45^{\circ}$ with respect to the principal axes of the strain rate tensor $($ denoted as $\nabla \tilde{\mathbf{u}})$. Then, $(\nabla \tilde{\mathbf{u}})_{S H}$ is computed with

$$
(\nabla \tilde{\mathbf{u}})_{S H}=\left[\begin{array}{cc}
0 & \frac{\partial \tilde{v}}{\partial \tilde{x}}-\operatorname{sgn}\left(\frac{\partial \tilde{v}}{\partial \tilde{x}}\right) \cdot \min \left(\left|\frac{\partial \tilde{u}}{\partial \tilde{y}}\right|,\left|\frac{\partial \tilde{v}}{\partial \tilde{x}}\right|\right)
\end{array} \quad \begin{array}{c}
\frac{\partial \tilde{u}}{\partial \tilde{y}}-\operatorname{sgn}\left(\frac{\partial \tilde{u}}{\partial \tilde{y}}\right) \cdot \min \left(\left|\frac{\partial \tilde{u}}{\partial \tilde{y}}\right|,\left|\frac{\partial \tilde{v}}{\partial \tilde{x}}\right|\right) \\
0
\end{array}\right] .
$$

The vorticity associated with the shear component of the velocity gradient tensor is calculated as $\omega_{S H}=(\nabla \tilde{\mathbf{u}})_{S H, 21}-(\nabla \tilde{\mathbf{u}})_{S H, 12}$, i.e., the element on the second row/first column minus the element on the first row/second column. An example of an instantaneous $\omega_{S H}$ distribution is presented in Figure 3. Shear layers are visible which separate large scale regions with nearly uniform velocity, similar to observations by Meinhart and Adrian. ${ }^{17}$ The shear layer like structure that is present inside the rectangle (indicated in Figure 3(a)) can directly be related to an instantaneous jump in the streamwise velocity component (Figure 3(b)). To distinguish between surrounding flow and intense shear layers, a composite threshold criterion is used. First of all, the $\omega_{S H}$ noise level is estimated as before in the non-turbulent part of the flow. Three times this level effectively separates noise from actual shear layers. However, close to the wall, the average shear is significantly higher compared to the outer region. Hence, in the near wall region, a threshold is set based on the mean shear vorticity $\left\langle\omega_{S H}\right\rangle$ to detect internal layers closer to the wall. The highest value of either is found to be the suitable criteria to detect internal layers throughout the boundary layer.

Next, the geometrical characteristics of the internal layers are quantified. It should be noted that all of the areas above a certain threshold, shown in Figure 3(a), are regarded as internal layers. This eliminates the possibility of deliberately favouring certain layers, thereby distorting the statistics. Of course, this comes at the cost of slightly biased statistics. A probability density function (PDF)
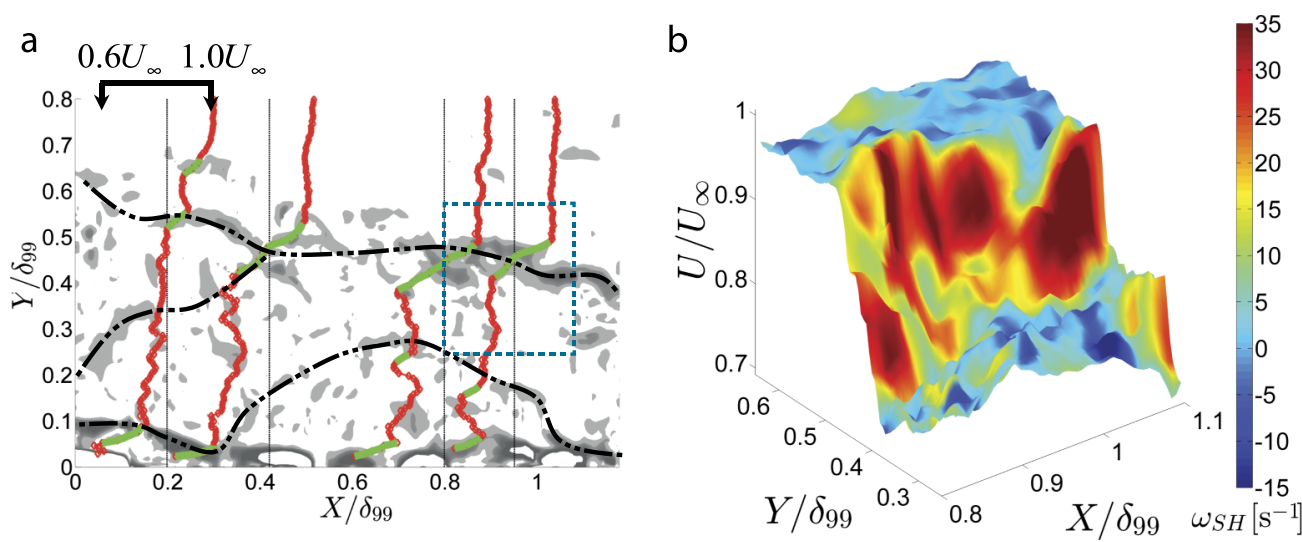

FIG. 3. (a) An instantaneous realization of the shear component of the vorticity $\omega_{S H}$, indicated with gray contours. The dashed dotted lines indicate the edges of uniform momentum zones determined from visual inspection of the velocity field. Streamwise velocity profiles are plotted to show the instantaneous jump across a shear layer. Regions of high velocity gradients are indicated with green dots. (b) Surface plot of the streamwise velocity field around the shear layer inside the rectangle in (a). For visualization purposes, this image has been Gaussian filtered to suppress noise, characterized by a standard deviation $\sigma / \delta_{99}=0.003$. 

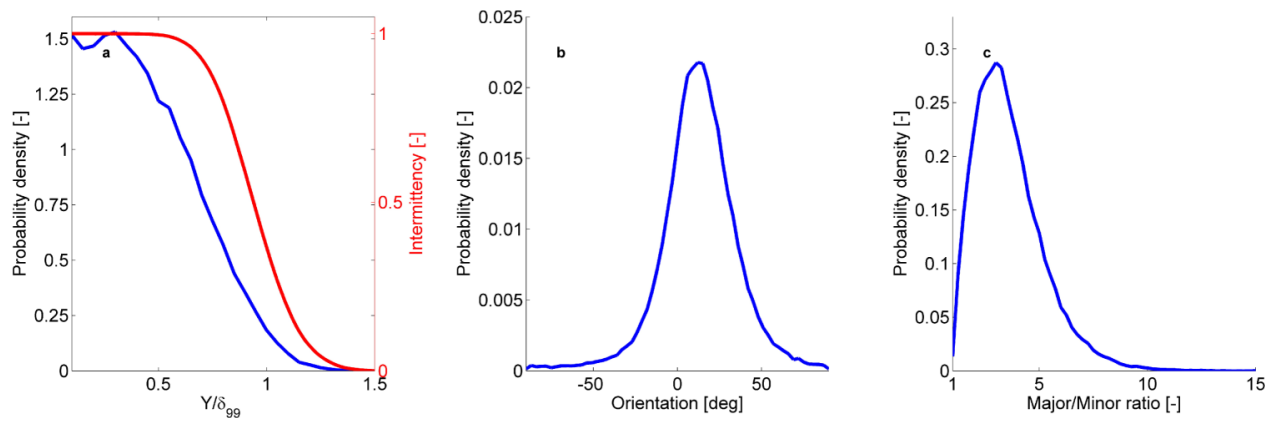

FIG. 4. (a) PDF of the centroidal location of the internal layers over the height of the TBL. The red curve depicts the intermittency curve determined from the T/NT interface. (b) PDF of the orientation of the internal layers with respect to the wall, present throughout the TBL. (c) PDF of the ratio between the major axis length and the minor axis length of internal layers, present throughout the TBL.

of the centroidal location is shown in Figure 4(a), together with the intermittency function obtained from the position of the T/NT interface. The occurrence of internal layers closely follows the intermittency profile, suggesting internal layers are distributed uniformly throughout the turbulent region of the flow. This behavior also indicates why the conditional profiles shown later in Sec. V in the outer region of the TBL do not appear to be converged. The number of detected internal layers is insufficient to obtain converged statistics in that part. The orientation with respect to the wall and the major axis length versus the minor axis of the internal layers are determined by fitting an ellipse with the same area moment of inertia as the detected layer, see Figures 4(b) and 4(c). It turns out that the average orientation of $12^{\circ}$ closely resembles the angle that Adrian et al. ${ }^{18}$ report for the angle of hairpin packets. This underlines once more that the effect of the pressure gradient in the current measurements is negligible as one of the first observable consequences of a favourable pressure gradient is to decrease the inclination of structures observed based on two-point correlations for $u^{\prime} .{ }^{31}$ Moreover, the angle of the shear layers remains relatively constant over the height of the boundary layer (not shown here). Finally, the PDF of the major/minor axis of the internal layers shows that the detected regions of high shear are elongated, i.e., layer-like structures, as their aspect ratio is on an average of 3.6.

\section{BOUNDARY ENTRAINMENT VELOCITY AND MODEL DESCRIPTION}

A short description of the model used to determine the boundary entrainment velocity is given in this section. The boundary entrainment velocity is defined here as the velocity with which the interface convects away from the wall, when following the interface in space (in a Lagrangian sense). Our approach corresponds with the definition of the boundary entrainment velocity $E_{b}$ as given by Westerweel et al. ${ }^{3}$ and Turner. ${ }^{37}$ The last reference clearly points out the different usages of the term entrainment velocity. A clear distinction should be made between the velocity with which the interface spreads outward $\left(E_{b}\right)$, the rate at which external fluid flows into the turbulent flow across a boundary $(E)$, and the outward velocity of the interface relative to the local mean flow $\left(E_{f}\right)$. For instance, in case of a turbulent jet, Turner ${ }^{37}$ predicted that the outward velocity at the edge of the jet measured in a fixed coordinate system will be $E_{b}=-2 E$. This was experimentally confirmed by Westerweel et al. ${ }^{3}$ by employing the super-layer jump condition. In order to derive the boundary entrainment velocity for the T/NT interface and the internal shear layers, a first order jump model is presently applied. This model is based on the work by VanZanten et al. ${ }^{38}$ and Pino et al. ${ }^{39}$ who used it to determine the growth rate of convective boundary layers. In a similar fashion, the spatial growth rate can be calculated for a neutral convective boundary layer as will be shown here. The reason not to choose a zeroth order jump model as proposed by Lilly ${ }^{40}$ is because his model assumes $\langle U\rangle$ to be discontinuous over an inversion, which is certainly not applicable when inspecting the conditional profiles (i.e., $\Delta\langle U\rangle$ occurs over a small but finite region $\delta_{w}$ ). In the current work, the situation as sketched in Figure 5 is considered. Consider the idealized profiles for the conditional streamwise 


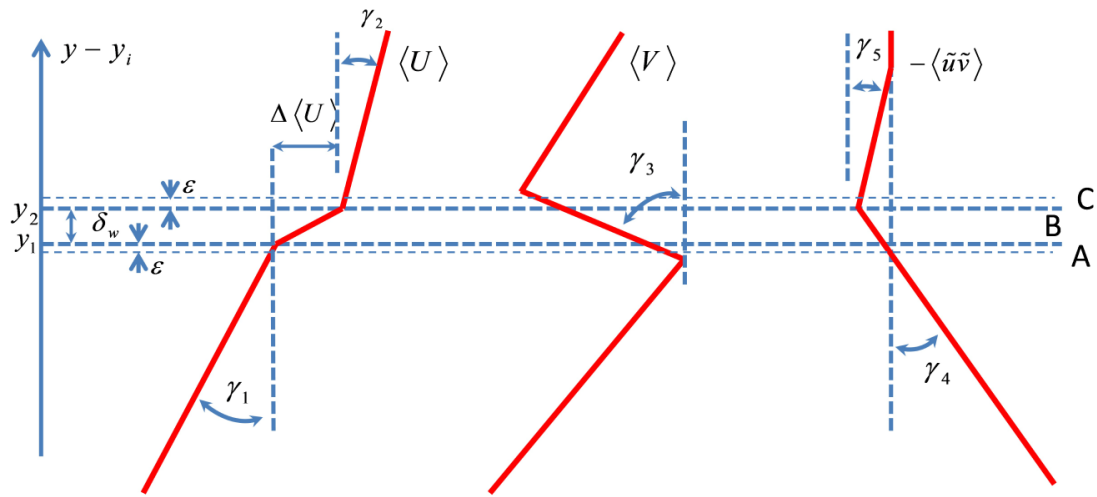

FIG. 5. Schematic description of the model. The average interface location is located between $y_{1}-y_{2}$. The jump in streamwise velocity occurs over a finite region $\delta_{w}$. The three integration regions are given by the letters A-C.

velocity $\langle U\rangle$, the conditional wall-normal velocity $\langle V\rangle$, and the conditional Reynolds shear stresses $\langle\tilde{u} \tilde{v}\rangle$ as given in Figure 5. The lowercases $\tilde{u}$ and $\tilde{v}$ denote the fluctuating velocity components with respect to the conditional mean velocities $\langle U\rangle$ and $\langle V\rangle$. In this model, the jump in streamwise velocity $\Delta\langle U\rangle$ occurs over a finite region $\delta_{w}$ (i.e., between $y_{1}-y_{2}$ ). Furthermore, on both sides of the interface, the conditional streamwise and Reynolds stress profiles show approximately constant gradients denoted by, respectively, $\gamma_{1,2}, \gamma_{4,5}$. Finally, the conditional wall-normal velocity has a constant gradient in the jump region $\gamma_{3}$.

The profiles shown in Figure 5 are entered into the conditional mean $\mathrm{x}$-momentum equation, which in absence of viscous effects and pressure gradient effects reads

$$
\frac{\partial\langle U\rangle}{\partial t}=-\langle V\rangle \frac{\partial\langle U\rangle}{\partial y}-\frac{\partial\langle\tilde{u} \tilde{v}\rangle}{\partial y} .
$$

The pressure gradient is neglected as independent pitot measurements indicate a negligible small acceleration parameter. In order to derive the growth rate of both internal layers and the T/NT interface, Eq. (3) is integrated over three regions as follows:

A. in a small region below the interface, between $\left(y_{1}-\epsilon\right)$ and $y_{1}$, where $\epsilon$ has a small but finite value;

B. over the jump region, between $y_{1}$ and $y_{2}$; and

C. in a small region above the interface, between $y_{2}$ and $\left(y_{2}+\epsilon\right)$.

By substitution of the results obtained from regions $\mathrm{A}$ and $\mathrm{C}$ into the equation obtained from region $B$, the boundary entrainment velocity is derived as

$$
\begin{aligned}
E_{b}=\frac{1}{\Delta\langle U\rangle-\frac{\delta_{w}}{2}\left(\gamma_{1}+\gamma_{2}\right)}\left[-\frac{\delta_{w}}{2}\left(\gamma_{1}\langle V\rangle_{y_{1}}+\gamma_{2}\langle V\rangle_{y_{2}}+\gamma_{4}+\gamma_{5}-\gamma_{3} \Delta\langle U\rangle\right)-\right. \\
\left.\langle\tilde{u} \tilde{v}\rangle_{y_{1}}+\langle\tilde{u} \tilde{v}\rangle_{y_{2}}+\Delta\langle U\rangle\langle V\rangle_{y_{1}}\right] .
\end{aligned}
$$

The complete derivation of Eq. (4) can be found in the Appendix.

\section{CONDITIONAL SAMPLING}

\section{A. T/NT interface}

Conditional sampling is performed at fixed wall-normal distances with respect to the location of the interface. This conditional sampling technique is described in detail in Bisset et al. ${ }^{33}$ The conditional profiles of the streamwise velocity, wall-normal velocity, Reynolds shear stress, and the out-of-plane vorticity $\Omega_{z}$ are shown in Figure 6, each normalized with appropriate large scale parameters. First of all, the conditional streamwise velocity profile indicates a distinct jump over the $\mathrm{T} / \mathrm{NT}$ interface of approximately $0.04 U_{\infty}$. In the turbulent region below the interface, this profile 

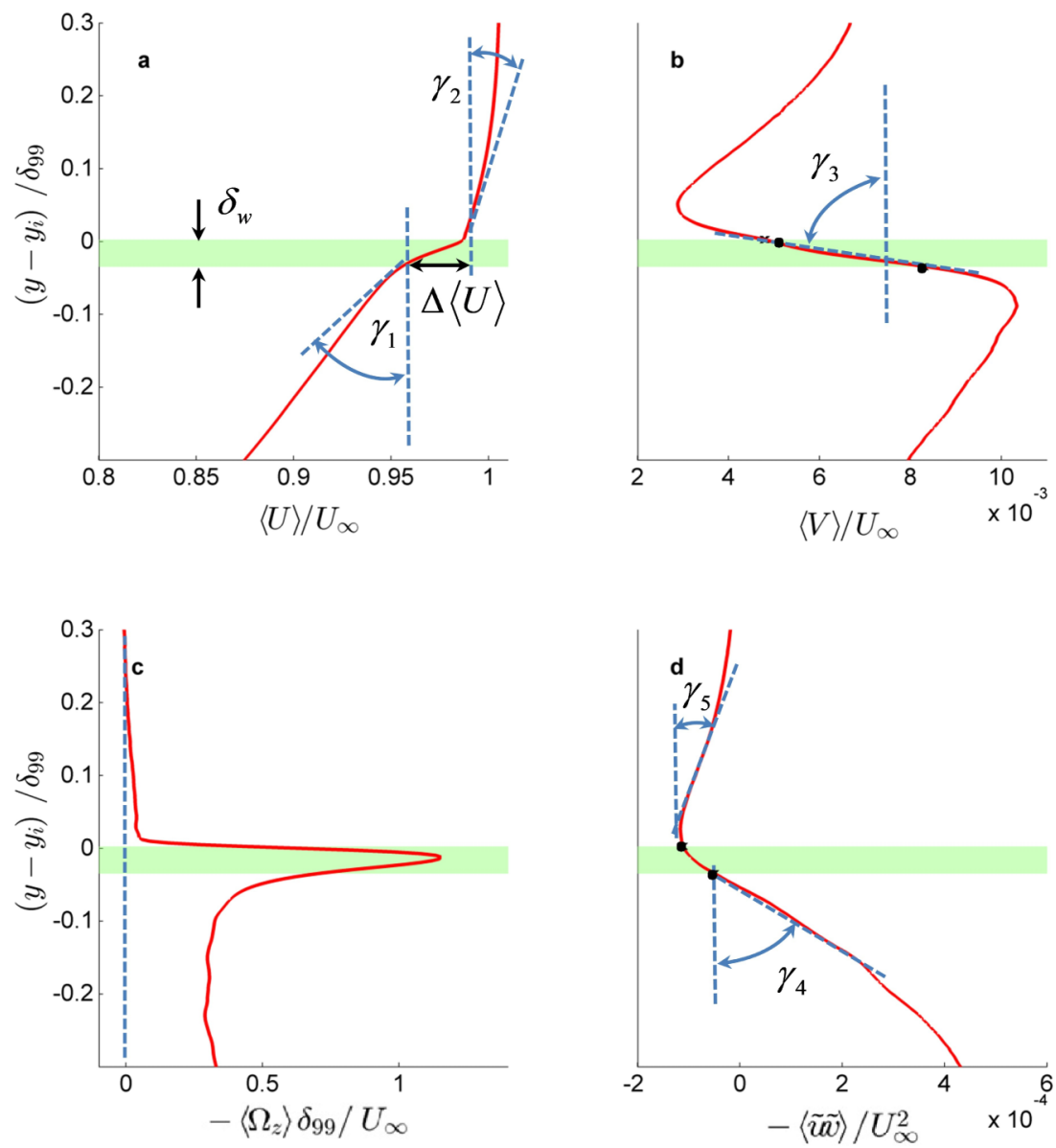

FIG. 6. (a) Conditionally sampled velocity profile $\langle U\rangle$ over the T/NT interface. (b) Conditional $\langle V\rangle$. (c) Conditional out-of-plane vorticity $\left\langle\Omega_{z}\right\rangle$. (d) Conditional sampled Reynolds shear stress profiles $\langle\tilde{u} \tilde{v}\rangle$. The green patch indicates the jump region. The relevant gradients are indicated by $\gamma_{1}-\gamma_{5}$. The values for $V_{y_{1}}, V_{y_{2}},\langle\tilde{u} \tilde{v}\rangle_{y_{2}}$, and $\langle\tilde{u} \tilde{v}\rangle_{y_{1}}$ are indicated by black dots. Positive y-axis points in the same direction as the wall-normal axis.

exhibits a constant gradient which is consistent with the approximately constant value for $\Omega_{z}$ in this region. Second, on the non-turbulent side of the interface, there is still a finite gradient in the conditional streamwise velocity. This behavior is probably due to the presence of irrotational fluctuations in the non-turbulent part of the flow. ${ }^{1,41}$ Furthermore, the conditional out-of-plane vorticity in the non-turbulent part quickly drops to zero, as shown in Figure 6(c). This confirms once more that the correct T/NT has been detected. The vorticity strongly peaks inside the T/NT interface and it reaches approximately a constant value on the turbulent side of the interface. This behavior is consistent with the observations from jets ${ }^{3}$ and TBL. ${ }^{12}$ From Figure 6(b), it is observed that the gradient in the conditional wall-normal velocity profile is negative over the interface. This wall-normal compression behavior implies that out-of-plane vorticity stretching occurs over this interface in order to maintain a sharp interface. Overall, it should be noted that the profiles shown in Figure 6 appear to be consistent with the model proposed in Figure 5.

In order to derive the entrainment velocity of the T/NT interface, the first order jump model as described in Sec. IV is employed. First of all, the edges of the T/NT interface are determined from the linear fits made to the velocity profile in Figure 6(a) on both the turbulent side (lower part) and the non-turbulent side (upper part). For the current research, the intersections between these fits are regarded as the edges of the T/NT interface; this yields a jump in streamwise velocity $\Delta\langle U\rangle=0.04 U_{\infty}$ and a thickness over which this jump occurs of $\delta_{w}=0.035 \delta_{99} . \delta_{w}$ is an important geometric attribute; it describes the characteristic "thickness" of mixing layer eddies that exchange 
momentum between zones of uniform momentum. The different gradients, depicted in Figure 5, that are required to calculate $E_{b}$ are indicated in Figure 6. The values for the wall-normal and the Reynolds shear stress at top and bottom side of the interface are indicated by black dots. Combining these results and substituting this into Eq. (4) produces a boundary entrainment velocity $E_{b}=0.0109 U_{\infty}$

A second validation strategy adopting a different approach is employed to calculate the boundary layer growth rate, using a method as described by White. ${ }^{42}$ Basically, von Kármán's momentum law $\left(c_{f}=2 \frac{d \theta}{d x}\right)$ and a power-law approximation to the friction number $\left(c_{f} \approx 0.02 R e_{\delta}^{-1 / 6}\right)$ are combined together with a $1 / 5 t h$ power law approximation of the TBL velocity profile. A fit to the mean velocity profile of the TBL (not shown here) justifies a $1 / n t h$ power law approximation to the mean velocity profile, where $n=0.1931 \pm 0.002$. Therefore, the $1 / 5 t h$ power law approximation (with a difference of less than $2 \%$ ) was applied to predict the global boundary layer growth. This yields a first order differential equation that can be solved by separation of variables. The spatial growth rate is thus estimated as

$$
\frac{d \delta}{d x} \approx 0.14 \frac{6}{7}\left(\frac{v}{U_{\infty} x}\right)^{\frac{1}{5}} \approx 0.0142
$$

where $x$ is taken as the distance behind the trip wire (i.e., $x=3.5 \mathrm{~m}$ ). The average T/NT interface position is observed at $\left\langle\delta_{\text {int }}\right\rangle \approx 0.90 \delta_{99}$. Hence, the spatial growth rate of the T/NT interface is approximated by $\frac{d \delta_{i n t}}{d x} \approx \frac{\delta_{\text {int }}}{\delta_{09}} \frac{d \delta}{d x} \approx 0.0128$. From the conditional streamwise velocity profile in Figure 6(a), it is observed that the local convection velocity at the mean interface location is $U_{c} \approx 0.97 U_{\infty}$. Hence, the boundary entrainment velocity can be approximated by

$$
E_{b} \approx U_{c} \frac{d \delta_{\text {int }}}{d x} \approx 0.0124 U_{\infty} .
$$

Given the above assumptions and the finite measurement precision, the difference between Eq. (6) and the results obtained from the first order jump model falls within acceptable limits. Comparing the result given in Eq. (6) and the results obtained from the first order jump model, it turns out that both are in close agreement (i.e., $\approx 12 \%$ difference). Hence, the analysis near the T/NT interface could be directly related to the large scale features of the boundary layer growth rate.

\section{B. Internal layers}

Conditional sampling is also performed on the internal layers. The resulting profiles of the streamwise velocity, wall-normal velocity, and the Reynolds shear stress are given in Figure 7. Comparing the conditional streamwise velocity profiles for the T/NT interface (Figure 6) and the internal shear layers (Figure 7), it is clear that both display qualitatively similar behavior. This supports the notion that the shear layers inside the TBL are in essence similar to the external T/NT interface in the current framework of the integral momentum balance approach. It should be noted however that this observation does not imply that both the T/NT interface and the internal shear layers are identical in the small scale details which have been lost in the conditional averaging process. The jump in conditional streamwise velocity shown in Figure 7(a) indicates a strong increase towards the wall, whereas an approximately constant velocity jump is obtained far away from the wall $\left(y / \delta_{99}>0.5\right)$. Furthermore, $\partial\langle U\rangle / \partial y$ on the bottom side of the internal layers increases much more rapidly compared to the top side. As such, the flux of momentum through the interface increases closer to the wall. From Figure $7(b)$, it is clear that $\frac{\partial\langle V\rangle}{\partial y}$ is negative over the internal layers similar to the external T/NT interface, indicative of vorticity stretching happens within these internal layers. It should be noted that the Reynolds shear stress profiles in the outer region of the TBL do not appear to be converged (Figure 7(c)). As is evident from Figure 4(a), it is due to the small number of layers found at that location.

Closer to the wall the conditional Reynolds shear stress profiles $\langle\tilde{u} \tilde{v}\rangle$, shown in Fig. 7(c), depict a distinct jump over the layer, which increases when approaching the wall. On the top side of the layers (i.e., $\left.\left(y-y_{i}\right) / \delta_{99}>0\right)$, the shear stress profiles steadily decrease towards zero. It should be noted that in contrast to the T/NT interface, the shear stresses over the internal layers never reach 

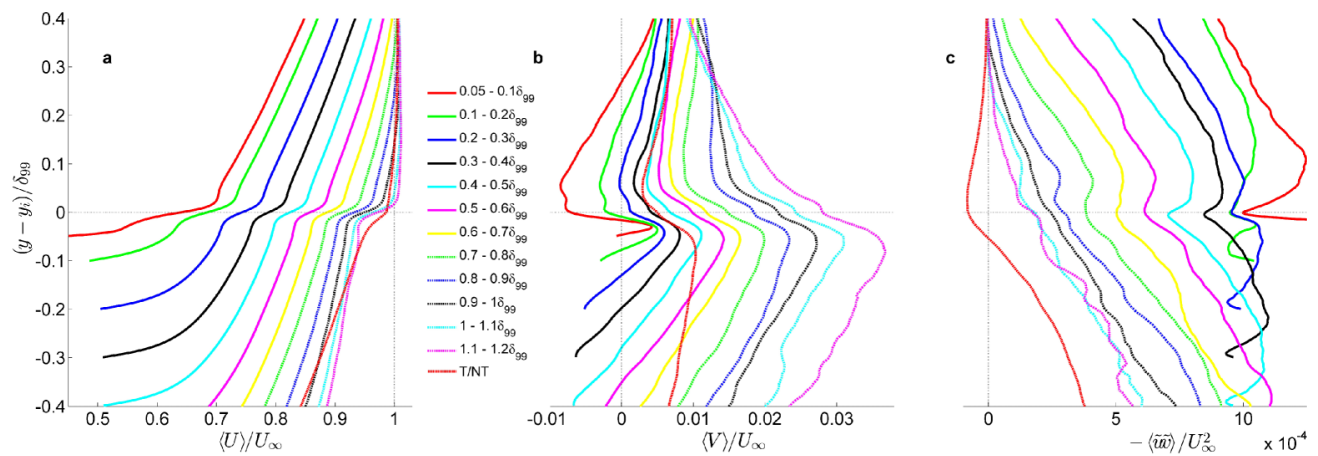

FIG. 7. (a) Conditionally sampled $\langle U\rangle$ velocity profiles over internal shear layers. (b) Conditional wall-normal velocity profiles. (c) Conditionally sampled Reynolds shear stress profiles. Positive y-axis points in the same direction as the wall-normal axis. Shear layers are binned according to their centroidal location in the boundary layer with a binsize of $0.1 \delta_{99}$.

positive values. On the bottom side of the shear layers (i.e., $\left.\left(y-y_{i}\right) / \delta_{99}<0\right)$, near wall peaks in Reynolds shear stress are observed (i.e., for layers below $y / \delta_{99}=0.5$ ).

The velocity jump across the internal layers is quantified in Figure 8(a). As mentioned before, an approximately constant value is observed for $y / \delta_{99}>0.5$ (Figure 8(a)). The jump thickness is observed to scale with the local Taylor microscale (Figure $8(\mathrm{~b}), \delta_{w} \approx 0.4 \lambda_{T}$ ), which is in accordance with the scaling of the T/NT interface thickness. ${ }^{7,10}$

As a next step, the model approach as shown in Figure 5 is applied to the internal layers to obtain $E_{b}$ for each internal layer from Eq. (4). The result is presented in Figure 9. A standard bootstrapping method is applied to derive the confidence interval. The first point above the wall indicates a large confidence interval due to the large gradients that are present. It turns out that the internal shear layers move faster away from the wall as $y / \delta_{99}$ increases. The conceptual picture that emerges from this graph is that one may have a TBL with multiple shear layers where the outermost layers move at a velocity faster than the innermost layers. It may well be that shear layers accelerate when moving up into the boundary layer and then eventually become the new T/NT interface as the old interface further from the wall dissipates and disappears. It should be noted that this concept only holds for the average shear layers, as instantaneously there still might be coalescence between different shear layers. Note that the entrainment velocities of the internal layers present in the outer layer (i.e., at $y / \delta_{99}=0.8-0.9$ ) are approximately twice as high as the boundary layer growth rate calculated before. Hence, internal shear layers have the tendency to run into the non-turbulent region faster than the overall growth of the boundary layer. Comparing the internal shear layer entrainment velocity $E_{b}$ with the wall normal velocity profile $V$ obtained from DNS data, ${ }^{43}$ it is observed that the shear layers are not moving with the average wall-normal velocity. In fact, the entrainment velocity of the internal shear layers $E_{b}$ is larger than $V$. This indicates that the layers are dynamically significant ${ }^{2}$ and there is actual mass transport across the layers.
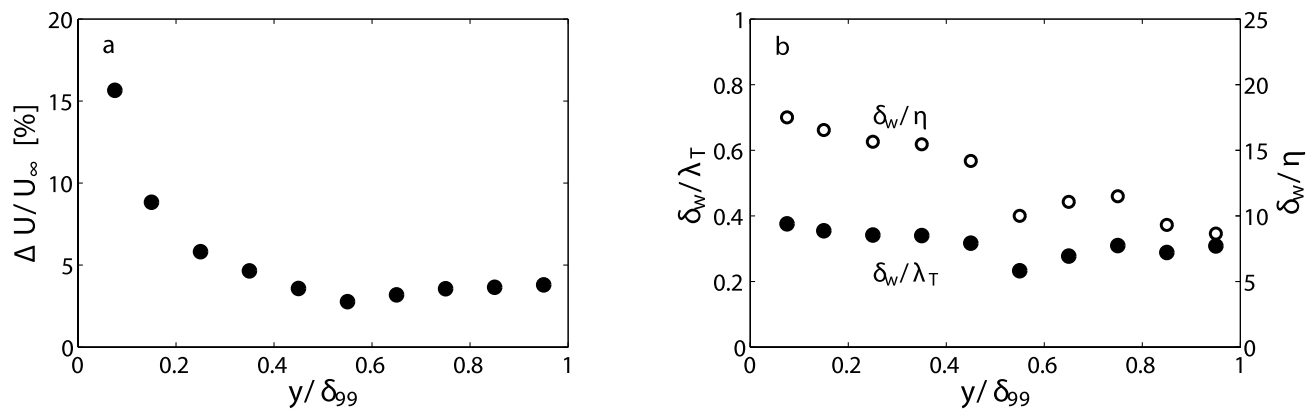

FIG. 8. (a) The streamwise velocity jump $\Delta U$ normalized with $U_{\infty}$ as function of the wall normal distance. (b) Layer thickness estimated from the streamwise velocity jump of the internal layers normalized with the local $\lambda_{T}$ and with the local Kolmogorov length scale $\eta$. 


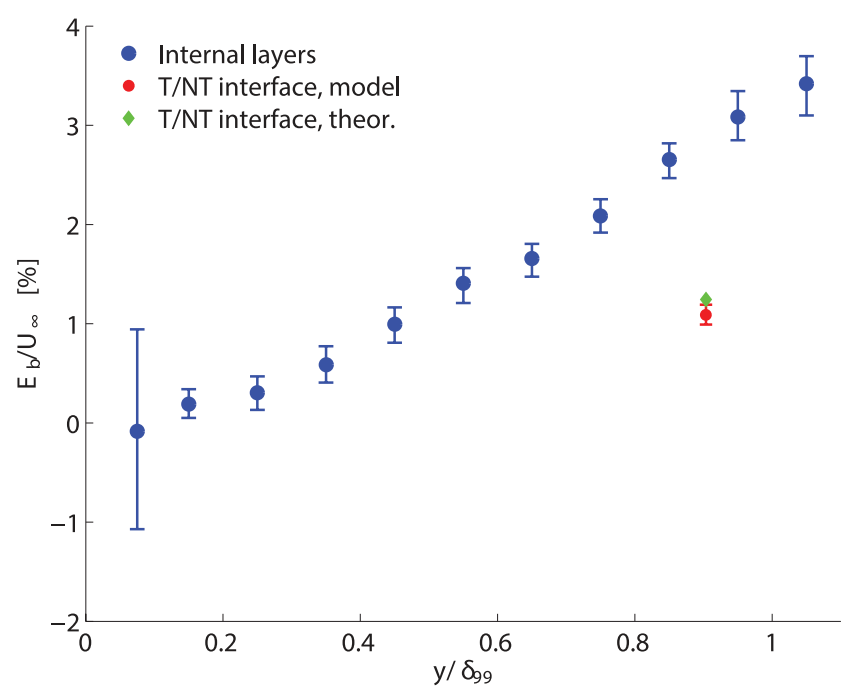

FIG. 9. The boundary entrainment velocity $E_{b}$ of the internal shear layers (blue dots) normalized with $U_{\infty}$ as function of the wall normal distance. The errorbar indicates the $95 \%$ confidence interval obtained from a standard bootstrapping method. The red dot indicates $E_{b}$ of the T/NT interface obtained from the first-order jump model, whereas the green dot indicates the $E_{b}$ for the T/NT interface obtained from Eq. (6).

\section{DISCUSSION}

As suggested in Figure 3, shear layers separate large scale regions of approximately uniform momentum. This has been observed before by Meinhart and Adrian, ${ }^{17}$ Adrian et al.,${ }^{18}$ Christensen and Adrian, ${ }^{23}$ Hambleton et al., ${ }^{21}$ and Wei et al. ${ }^{25}$ From the analysis performed in Sec. V, we derived the entrainment velocities of conditionally averaged shear layers. This process can be regarded as analogous to the overall boundary layer entrainment by the T/NT interface, which governs the growth of the TBL. A first suggestion that presents itself is that the entrainment velocities of the shear layers may be indicative for the growth rate of the uniform momentum zones that they bound. This is supported by the observation that the angle of the internal shear layers is found to be $12^{\circ}$, which corresponds to the typical angle of hairpin packets. ${ }^{18}$ From Figure 9, it is clear that the conditionally averaged shear layers in the region $y / \delta_{99}>0.5$ move at velocities on the order of the average boundary layer growth rate, shown as the red/green dot. We therefore conjecture that large scale regions on average grow slowly, i.e., with the same order of magnitude as the average boundary layer growth rate. Furthermore, it is worth mentioning that the boundary entrainment velocity of the average shear layer at $y / \delta_{99}=0.2\left(E_{b} \approx 0.003 U_{\infty}\right)$ shows a correspondence with the work of Elsinga et al. ${ }^{44}$ who found the average wall-normal convection velocity of spanwise vortices to be $0.006 U_{e} \pm 0.002 U_{e}$. Assuming these vortices populate the shear layers, this correspondence with the motion of instantaneous vortex structures supports the present conditional averaging approach. Furthermore, the recent work by Zheng and Longmire ${ }^{45}$ showed that $77 \%$ of the observed low momentum regions remained coherent over a significant long streamwise distance $\left(>9 \delta_{99}\right)$. Similar results have been obtained by Dennis and Nickels. ${ }^{46}$ As such, the layers accompanying these large scale motions will also be persistent over long streamwise distances. A simple analysis using Figures 9 and 1(a) is carried out in order to estimate where the average shear layer at a height $y / \delta_{99}=0.95$ may have originated from. First, the movement of the shear layer is evaluated in the wall-normal direction based on its entrainment velocity (Figure 9), yielding its y-position with time $y^{*}(t)$ between $y=0$ and $y=0.95 \delta_{99}$. At the same time, the layer also travels a distance $x^{*}$ in the streamwise direction, which is obtained by integrating the mean velocity profile over $y^{*}(t)$. Based on this calculation, it turns out that this average layer originated from the wall approximately $50 \delta_{99}$ upstream. As we measured $40 \delta_{99}$ downstream of the boundary layer trip, this cannot be the case. Therefore, we conjecture that shear layers may be generated not only at the wall but also via distinct (yet unknown) mechanisms away from the wall. This concept challenges the idea of, for 
instance, Head and Bandyopadhyay. ${ }^{47}$ Based on their visualization study, they proposed that hairpin structures are generated at the wall and rapidly grow into the outer region of the turbulent boundary layer.

\section{SUMMARY AND CONCLUSIONS}

In this paper, the characteristics of the T/NT interface and the internal layers have been quantified experimentally in a turbulent boundary layer. Layered structures have been observed before in statistical analysis of different turbulent flows. ${ }^{23,24}$ However, the current results confirm the existence of such layered structures in instantaneous flow fields. Conditional sampling is used to determine the quantitative properties of these layers. Compelling evidence is given that the internal layers and the external T/NT interface show qualitatively the same behavior in terms of the conditional velocity profiles. A first order jump model is applied in order to estimate the entrainment velocities of the interface and internal layers. By this approach, a correct prediction of the overall boundary layer growth could be obtained. Furthermore, conditional averaged internal layers move slowly in close vicinity of the wall, whereas their entrainment velocity is of the order of the boundary layer growth rate in the outer part of the TBL. The internal layers are believed to bound the large scale motions in the flow. Therefore, the current results strongly suggest that these large scale motions only grow slowly, i.e., on the order of the boundary layer growth rate.

\section{ACKNOWLEDGMENTS}

The assistance of E.F.J. Overmars and Dr. Y. Jodai during the experiments and the fruitful discussions with Professor J.C.R. Hunt FRS are greatly acknowledged. The authors thank the Dutch Technology Foundation STW for their financial support.

\section{APPENDIX: THEORETICAL DERIVATION OF FIRST ORDER JUMP MODEL}

In this appendix, the theoretical derivation of the first order jump model as proposed by VanZanten $e t a l . .^{38}$ and Pino et al. ${ }^{39}$ is given with modifications for the internal shear layers. It starts by integrating mean x-momentum equation (3) over the distinct regions shown in Figure 5.

\section{Integrate over region $A$}

First, we integrate conditional momentum equation (3) over the region A which extends from $\left(y_{1}-\epsilon\right)$ to $y_{1}$ below the layer,

$$
\int_{y_{1}-\epsilon}^{y_{1}} \frac{\partial\langle U\rangle}{\partial t} \mathrm{~d} y=-\int_{y_{1}-\epsilon}^{y_{1}}\langle V\rangle \frac{\partial\langle U\rangle}{\partial y} \mathrm{~d} y-\int_{y_{1}-\epsilon}^{y_{1}} \frac{\partial\langle\tilde{u} \tilde{v}\rangle}{\partial y} \mathrm{~d} y .
$$

Then, we apply the Leibniz integral rule to the left hand side (LHS),

$$
\begin{aligned}
\int_{y_{1}-\epsilon}^{y_{1}} \frac{\partial\langle U\rangle}{\partial t} \mathrm{~d} y & =\frac{\partial}{\partial t} \int_{y_{1}-\epsilon}^{y_{1}}\langle U\rangle \mathrm{d} y-\langle U\rangle_{y_{1}} \frac{\partial y_{1}}{\partial t}+\left[\langle U\rangle_{y_{1}}-\gamma_{1} \epsilon\right] \frac{\partial\left(y_{1}-\epsilon\right)}{\partial t} \\
& =\frac{\partial}{\partial t}\left[\epsilon\langle U\rangle_{y_{1}}-\frac{1}{2} \epsilon^{2} \gamma_{1}\right]-\epsilon \gamma_{1} \frac{\partial y_{1}}{\partial t},
\end{aligned}
$$

where it is assumed that the gradient of the streamwise conditional velocity $\gamma_{1}=\left.\frac{\partial\langle U\rangle}{\partial y}\right|_{y<y_{1}}$ is constant in this region and $\frac{\partial\left(y_{1}-\epsilon\right)}{\partial t}=\frac{\partial\left(y_{1}\right)}{\partial t}$, because $\epsilon$ is constant. The first term on the right hand side (RHS) of Eq. (A1) can be written as

$$
\begin{aligned}
-\int_{y_{1}-\epsilon}^{y_{1}}\langle V\rangle \frac{\partial\langle U\rangle}{\partial y} \mathrm{~d} y & =-\gamma_{1} \int_{y_{1}-\epsilon}^{y_{1}}\langle V\rangle \mathrm{d} y \\
& =-\epsilon \gamma_{1}\langle V\rangle_{y_{1}},
\end{aligned}
$$


where again it is assumed that the gradient of the conditional streamwise velocity profile is constant (i.e., $\gamma_{1}$ ). The last term on the RHS of Eq. (A1) can be written as

$$
-\int_{y_{1}-\epsilon}^{y_{1}} \frac{\partial\langle\tilde{u} \tilde{v}\rangle}{\partial y} \mathrm{~d} y=-\epsilon \gamma_{4}
$$

where $\gamma_{4}=\left.\frac{\partial\langle\tilde{u} \tilde{v}\rangle}{\partial y}\right|_{y<y_{1}}$ is the gradient in the Reynolds stress in the turbulent region below the interface. Combining these results yields

$$
\frac{\partial}{\partial t}\left[\epsilon\langle U\rangle_{y_{1}}-\frac{1}{2} \epsilon^{2} \gamma_{1}\right]-\epsilon \gamma_{1} \frac{\partial y_{1}}{\partial t}=-\epsilon \gamma_{1}\langle V\rangle_{y_{1}}-\epsilon \gamma_{4} .
$$

Rearranging Eq. (A5), dividing by $\epsilon$, and taking the limit of $\epsilon \rightarrow 0$ yield

$$
\frac{\partial\langle U\rangle_{y_{1}}}{\partial t}=\gamma_{1}\left(\frac{\partial y_{1}}{\partial t}-\langle V\rangle_{y_{1}}\right)-\gamma_{4}
$$

\section{Integrate over region $B$}

Next, we integrate Eq. (3) over the jump region B (i.e., $y_{1}<y<y_{2}$ ),

$$
\int_{y_{1}}^{y_{2}} \frac{\partial\langle U\rangle}{\partial t} \mathrm{~d} y=-\int_{y_{1}}^{y_{2}}\langle V\rangle \frac{\partial\langle U\rangle}{\partial y} \mathrm{~d} y-\int_{y_{1}}^{y_{2}} \frac{\partial\langle\tilde{u} \tilde{v}\rangle}{\partial y} \mathrm{~d} y .
$$

Now, Leibniz rule is applied to move the partial derivative of the LHS out of the integral. For the LHS of Eq. (A7), this yields

$$
\begin{aligned}
\int_{y_{1}}^{y_{2}} \frac{\partial\langle U\rangle}{\partial t} \mathrm{~d} y & =\frac{\partial}{\partial t} \int_{y_{1}}^{y_{2}}\langle U\rangle \mathrm{d} y-\langle U\rangle_{y_{2}} \frac{\partial y_{2}}{\partial t}+\langle U\rangle_{y_{1}} \frac{\partial y_{1}}{\partial t} \\
& =\frac{\partial}{\partial t}\left[\langle U\rangle_{y_{1}} \delta_{w}+\frac{\delta_{w}}{2} \Delta\langle U\rangle\right]-\Delta\langle U\rangle \frac{\partial y_{2}}{\partial t},
\end{aligned}
$$

where the assumption $\frac{\partial y_{2}}{\partial t} \approx \frac{\partial y_{1}}{\partial t}$ has been made. The first term on the RHS of Eq. (A7) is rewritten by using integration by parts and assuming that $\langle V\rangle$ is linear inside the jump region. In this region, $\langle V\rangle$ is approximated as $\langle V(y)\rangle=\langle V\rangle_{y_{1}}+\gamma_{3}\left(y-y_{1}\right)$, where $\gamma_{3}=\left.\frac{\partial\langle V\rangle}{\partial y}\right|_{y_{1}<y<y_{2}}$. Substituting this relation yields

$$
\begin{aligned}
-\int_{y_{1}}^{y_{2}}\langle V\rangle \frac{\partial\langle U\rangle}{\partial y} \mathrm{~d} y & =-\int_{y_{1}}^{y_{2}}\left(\langle V\rangle_{y_{1}}+\gamma_{3}\left(y-y_{1}\right)\right) \frac{\partial\langle U\rangle}{\partial y} \mathrm{~d} y \\
& =-\left[\left(\langle V\rangle_{y_{1}}+\gamma_{3}\left(y-y_{1}\right)\right)\langle U\rangle\right]_{y_{1}}^{y_{2}}+\int_{y_{1}}^{y_{2}}\langle U\rangle \frac{\partial\langle V\rangle}{\partial y} \mathrm{~d} y \\
& =-\Delta\langle U\rangle\langle V\rangle_{y_{1}}-\frac{1}{2} \gamma_{3} \delta_{w} \Delta\langle U\rangle
\end{aligned}
$$

where $\Delta\langle U\rangle$ is the jump in streamwise velocity over the layer and $\delta_{w}$ is the thickness of the jump region. The second term on the RHS of Eq. (A7) yields

$$
-\int_{y_{1}}^{y_{2}} \frac{\partial\langle\tilde{u} \tilde{v}\rangle}{\partial y} \mathrm{~d} y=-\langle\tilde{u} \tilde{v}\rangle_{y_{2}}+\langle\tilde{u} \tilde{v}\rangle_{y_{1}} .
$$

Combining the results from Eqs. (A8)-(A10) into Eq. (A7) yields

$$
\begin{aligned}
\delta_{w} \frac{\partial}{\partial t}\left[\langle U\rangle_{y_{1}}+\frac{1}{2} \Delta\langle U\rangle\right]= & \langle\tilde{u} \tilde{v}\rangle_{y_{1}}-\langle\tilde{u} \tilde{v}\rangle_{y_{2}}-\Delta\langle U\rangle\langle V\rangle_{y_{1}}-\frac{1}{2} \gamma_{3} \delta_{w} \Delta\langle U\rangle \\
& +\Delta\langle U\rangle \frac{\partial y_{2}}{\partial t} .
\end{aligned}
$$




\section{Integrate over region C}

The last integration step is performed on the non-turbulent side over region $\mathrm{C}$, between $y_{2}$ and $y_{2}+\epsilon$,

$$
\int_{y_{2}}^{y_{2}+\epsilon} \frac{\partial\langle U\rangle}{\partial t} \mathrm{~d} y=-\int_{y_{2}}^{y_{2}+\epsilon}\langle V\rangle \frac{\partial\langle U\rangle}{\partial y} \mathrm{~d} y-\int_{y_{2}}^{y_{2}+\epsilon} \frac{\partial\langle\tilde{u} \tilde{v}\rangle}{\partial y} \mathrm{~d} y .
$$

Using Leibniz once more for the LHS of Eq. (A12) yields

$$
\int_{y_{2}}^{y_{2}+\epsilon} \frac{\partial\langle U\rangle}{\partial t} \mathrm{~d} y=\frac{\partial}{\partial t}\left[\langle U\rangle_{\delta_{i}} \epsilon+\Delta\langle U\rangle \epsilon+\frac{\epsilon^{2}}{2} \gamma_{2}\right]-\epsilon \gamma_{2} \frac{\partial h}{\partial t},
$$

where the gradient of the conditional streamwise velocity is given as $\gamma_{2}=\left.\frac{\partial\langle U\rangle}{\partial y}\right|_{y>y_{2}}$. Assuming additionally that $\gamma_{5}=\left.\frac{\partial\langle\tilde{u} \tilde{v}\rangle}{\partial y}\right|_{y>y_{2}}$ is constant in the non-turbulent region, the RHS of Eq. (A12) is evaluated as

$$
-\int_{y_{2}}^{y_{2}+\epsilon}\langle V\rangle \frac{\partial\langle U\rangle}{\partial y} \mathrm{~d} y-\int_{y_{2}}^{y_{2}+\epsilon} \frac{\partial\langle\tilde{u} \tilde{v}\rangle}{\partial y} \mathrm{~d} y=-\epsilon \gamma_{2}\langle V\rangle_{y_{2}}-\epsilon \gamma_{5} .
$$

Substituting (A13) and (A14) into Eq. (A12), dividing by $\epsilon$, and taking the limit $\epsilon \rightarrow 0$ finally yield

$$
\frac{\partial}{\partial t}\left[\langle U\rangle_{y_{1}}+\Delta\langle U\rangle\right]=\gamma_{2}\left(\frac{\partial y_{2}}{\partial t}-\langle V\rangle_{h}\right)-\gamma_{5}
$$

\section{Calculating the growth rate of the boundary layer}

In order to determine the growth rate of the BL, different equations derived in Subsections 1-3 of the Appendix will be combined. First of all, the LHS of Eq. (A11) is rewritten as

$$
\delta_{w} \frac{\partial}{\partial t}\left[\langle U\rangle_{y_{1}}+\frac{1}{2} \Delta\langle U\rangle\right]=\frac{\delta_{w}}{2}\left[\frac{\partial\langle U\rangle_{y_{1}}}{\partial t}+\frac{\partial\left(\langle U\rangle_{y_{1}}+\Delta\langle U\rangle\right)}{\partial t}\right] .
$$

Now, Eqs. (A5) and (A15) can be substituted into Eq. (A11) yielding

$$
\begin{aligned}
\frac{\delta_{w}}{2}\left[\gamma_{1}\left(\frac{\partial y_{1}}{\partial t}-\langle V\rangle_{y_{1}}\right)-\gamma_{4}+\gamma_{2}\left(\frac{\partial y_{2}}{\partial t}-\langle V\rangle_{y_{2}}\right)-\gamma_{5}\right]=\cdots \\
\langle\tilde{u} \tilde{v}\rangle_{y_{1}}-\langle\tilde{u} \tilde{v}\rangle_{y_{2}}-\Delta\langle U\rangle\langle V\rangle_{y_{1}}-\frac{1}{2} \gamma_{3} \delta_{w} \Delta\langle U\rangle+\Delta\langle U\rangle \frac{\partial y_{2}}{\partial t},
\end{aligned}
$$

rewriting this equation to solve for $\frac{\partial y_{2}}{\partial t}$ yields

$$
\begin{aligned}
E_{b}=\frac{1}{\Delta\langle U\rangle-\frac{\delta_{w}}{2}\left(\gamma_{1}+\gamma_{2}\right)}\left[-\frac{\delta_{w}}{2}\left(\gamma_{1}\langle V\rangle_{y_{1}}+\gamma_{2}\langle V\rangle_{y_{2}}+\gamma_{4}+\gamma_{5}-\gamma_{3} \Delta\langle U\rangle\right)-\right. \\
\left.\langle\tilde{u} \tilde{v}\rangle_{y_{1}}+\langle\tilde{u} \tilde{v}\rangle_{y_{2}}+\Delta\langle U\rangle\langle V\rangle_{y_{1}}\right],
\end{aligned}
$$

where it is assumed that $E_{b}=\frac{\partial y_{2}}{\partial t} \approx \frac{\partial y_{1}}{\partial t}$, i.e., the jump thickness does not change with time. It is good to realize that once the jump thickness decreases to zero, the standard $0 t h$ order jump model is retrieved that was originally derived by Lilly. ${ }^{40}$

${ }^{1}$ S. Corssin and A. L. Kistler, "Free-stream boundaries of turbulent flows," NACA Technical Note 1244, 1955, pp. $1033-1064$.

2 J. C. R. Hunt, I. Eames, C. B. da Silva, and J. Westerweel, "Interfaces and inhomogeneous turbulence," Philos. Trans. R. Soc., A 369, 811-832 (2011).

${ }^{3}$ J. Westerweel, C. Fukushima, J. M. Pedersen, and J. C. R. Hunt, "Momentum and scalar transport at the turbulent/nonturbulent interface of a jet," J. Fluid Mech. 631, 199-229 (2009).

${ }^{4}$ J. Westerweel, C. Fukushima, J. M. Pedersen, and J. C. R. Hunt, "Mechanics of the turbulent-nonturbulent interface of a jet," Phys. Rev. Lett. 95, 174501 (2005).

5 T. Ishihara, T. Gotoh, and Y. Kaneda, "Study of high Reynolds number isotropic turbulence by direct numerical simulation," Annu. Rev. Fluid Mech. 41, 165-180 (2009). 
${ }^{6}$ T. Ishihara, Y. Kaneda, and J. C. R. Hunt, “Thin shear layers in high Reynolds number turbulence—DNS results," Flow, Turbul. Combust. 91, 895-929 (2013).

${ }^{7}$ C. B. da Silva and R. R. Taveira, "The thickness of the turbulent/nonturbulent interface is equal to the radius of the large vorticity structures near the edge of the shear layer," Phys. Fluids 22, 1-4 (2010).

${ }^{8}$ M. Holzner, A. Liberzon, M. Guala, A. Tsinober, and W. Kinzelbach, "Generalized detection of a turbulent front generated by an oscillating grid," Exp. Fluids 41, 711-719 (2006).

${ }^{9}$ M. Holzner, A. Liberzon, N. Nikitin, W. Kinzelbach, and A. Tsinober, "Small-scale aspects of flows in proximity of the turbulent/nonturbulent interface," Phys. Fluids 19, 071702 (2007).

${ }^{10}$ C. B. da Silva, J. C. R. Hunt, I. Eames, and J. Westerweel, "Interfacial layers between regions of different turbulence intensity,” Annu. Rev. Fluid Mech. 46, 567-590 (2014).

${ }^{11}$ M. van Reeuwijk and M. Holzner, “The turbulence boundary of a temporal jet,” J. Fluid Mech. 739, 254-275 (2013).

${ }^{12}$ K. Chauhan, J. Philip, C. M. de Silva, N. Hutchins, and I. Marusic, “The turbulent/non-turbulent interface and entrainment in a boundary layer," J. Fluid Mech. 742, 1-33 (2014).

${ }^{13}$ T. Ishihara, H. Ogasawara, and J. C. R. Hunt, "Analysis of conditional statistics obtained near the turbulent/non-turbulent interface of turbulent boundary layers," J. Fluids Struct. 53, 50-57 (2014).

${ }^{14}$ M. Iovieno, S. Di Savino, L. Gallana, and D. Tordella, "Mixing of a passive scalar across a thin shearless layer: Concentration of intermittency on the sides of the turbulent interface," J. Turbul. 15, 311-334 (2014).

${ }^{15}$ S. K. Robinson, "Coherent motions in the turbulent boundary layer," Annu. Rev. Fluid Mech. 23, 601-639 (1991).

${ }^{16}$ R. F. Blackwelder and L. S. G. Kovasznay, "Time scales and correlations in a turbulent boundary layer," Phys. Fluids 15, 1545-1554 (1972).

${ }^{17}$ C. D. Meinhart and R. J. Adrian, "On the existence boundary layer of uniform momentum zones in a turbulent boundary layer," Phys. Fluids 7, 694-696 (1995).

${ }^{18}$ R. J. Adrian, C. D. Meinhart, and C. D. Tomkins, "Vortex organization in the outer region of the turbulent boundary layer," J. Fluid Mech. 422, 1-54 (2000).

${ }^{19} \mathrm{~N}$. Hutchins and I. Marusic, "Evidence of very long meandering features in the logarithmic region of turbulent boundary layers," J. Fluid Mech. 579, 1-28 (2007).

${ }^{20}$ I. Marusic, B. J. McKeon, P. A. Monkewitz, H. M. Nagib, A. J. Smits, and K. R. Sreenivasan, "Wall-bounded turbulent flows at high Reynolds numbers: Recent advances and key issues," Phys. Fluids 22, 065103 (2010).

${ }^{21}$ W. T. Hambleton, N. Hutchins, and I. Marusic, "Simultaneous orthogonal-plane particle image velocimetry measurements in a turbulent boundary layer," J. Fluid Mech. 560, 53 (2006).

${ }^{22}$ B. Ganapathisubramani, E. K. Longmire, and I. Marusic, "Characteristics of vortex packets in turbulent boundary layers," J. Fluid Mech. 478, 35-46 (2003).

${ }^{23}$ K. T. Christensen and R. J. Adrian, "Statistical evidence of hairpin vortex packets in wall turbulence," J. Fluid Mech. 431, 433-443 (2001).

${ }^{24}$ G. E. Elsinga and I. Marusic, “Universal aspects of small-scale motions in turbulence,” J. Fluid Mech. 662, 514-539 (2010).

${ }^{25}$ L. Wei, G. E. Elsinga, G. Brethouwer, P. Schlatter, and A. V. Johansson, "Universality and scaling phenomenology of small-scale turbulence in wall-bounded flows," Phys. Fluids 26, 035107 (2014).

${ }^{26}$ M. Stanislas, L. Perret, and J. M. Foucaut, "Vortical structures in the turbulent boundary layer: A possible route to a universal representation," J. Fluid Mech. 602, 327-382 (2008).

27 J. Westerweel and F. Scarano, "Universal outlier detection for PIV data," Exp. Fluids 39, 1096-1100 (2005).

${ }^{28}$ C. Poelma, J. Westerweel, and G. Ooms, "Turbulence statistics from optical whole-field measurements in particle-laden turbulence," Exp. Fluids 40, 347-363 (2005).

${ }^{29}$ P. S. Klebanoff, "Characteristics of turbulence in a boundary layer with zero pressure gradient," NACA Technical Note 1247, 1951, pp. 1135-1153.

${ }^{30}$ A. Schröder, R. Geisler, K. Staack, G. E. Elsinga, F. Scarano, B. Wieneke, A. Henning, C. Poelma, and J. Westerweel, "Eulerian and Lagrangian views of a turbulent boundary layer flow using time-resolved tomographic PIV," Exp. Fluids 50, 1071-1091 (2010).

${ }^{31}$ P. Joshi, X. Liu, and J. Katz, "Effect of mean and fluctuating pressure gradients on boundary layer turbulence," J. Fluid Mech. 748, 36-84 (2014).

${ }^{32}$ D. B. DeGraaff and J. K. Eaton, "Reynolds-number scaling of the flat-plate turbulent boundary layer," J. Fluid Mech. 422, 319-346 (2000).

${ }^{33}$ D. K. Bisset, J. C. R. Hunt, and M. M. Rogers, “The turbulent/non-turbulent interface bounding a far wake,” J. Fluid Mech. 451, 383-410 (2002).

${ }^{34}$ J. M. Foucaut and M. Stanislas, "Some considerations on the accuracy and frequency response of some derivative filters applied to particle image velocimetry vector fields," Meas. Sci. Technol. 13, 1058-1071 (2002).

${ }^{35}$ V. Kolář, "Vortex identification: New requirements and limitations," Int. J. Heat Fluid Flow 28, 638-652 (2007).

${ }^{36}$ Y. Maciel, M. Robitaille, and S. Rahgozar, "A method for characterizing cross-sections of vortices in turbulent flows," Int. J. Heat Fluid Flow 37, 177-188 (2012).

${ }^{37}$ J. S. Turner, "Turbulent entrainment: The development of the entrainment assumption, and its application to geophysical flows," J. Fluid Mech. 173, 431-471 (1986).

${ }^{38}$ M. C. VanZanten, P. G. Duynkerke, and J. W. M. Cuijpers, "Entrainment parameterization in convective boundary layers," J. Atmos. Sci. 56, 813-828 (1999).

${ }^{39}$ D. Pino, J. Vila-Guerau de Arellano, and S. W. Kim, "Representing sheared convective boundary layer by zeroth- and first-order-jump mixed-layer models: Large-eddy simulation verification,” J. Appl. Meteorol. Climatol. 45, 1224-1243 (2006).

${ }^{40}$ D. K. Lilly, "Cloud-topped mixed layers under a strong inversion,” Q. J. R. Meteorol. Soc. 94, 292-309 (1968).

${ }^{41}$ O. M. Phillips, "The irrotational motion outside a free turbulent boundary," Math. Proc. Cambridge Philos. Soc. 51, 220-229 (1954). 
${ }^{42}$ F. M. White, Viscous Fluid Flow (McGraw-Hill Higher Education, 2006).

${ }^{43}$ G. Borrell, J. A. Sillero, and J. Jiménez, "A code for direct numerical simulation of turbulent boundary layers at high Reynolds numbers in BG/P supercomputers," Comput. Fluids 80, 37-43 (2013).

${ }^{44}$ G. E. Elsinga, C. Poelma, A. Schröder, R. Geisler, F. Scarano, and J. Westerweel, "Tracking of vortices in a turbulent boundary layer," J. Fluid Mech. 697, 273-295 (2012).

${ }^{45}$ S. Zheng and E. K. Longmire, "Perturbing vortex packets in a turbulent boundary layer," J. Fluid Mech. 748, 368-398 (2014).

${ }^{46}$ D. J. C. Dennis and T. B. Nickels, "On the limitations of Taylor's hypothesis in constructing long structures in a turbulent boundary layer," J. Fluid Mech. 614, 197 (2008)

${ }^{47}$ M. R. Head and P. Bandyopadhyay, "New aspects of turbulent boundary-layer structure," J. Fluid Mech. 107, 297-338 (1981). 